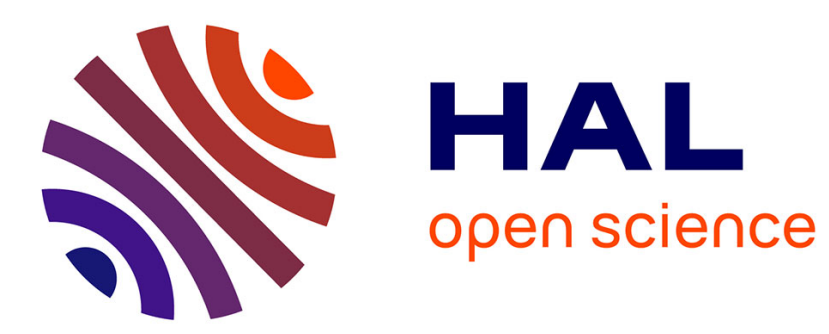

\title{
Calorimètre adiabatique à température variable et à surfaces d'échanges sphériques
}

\author{
R. Mainard, J. Kleinclauss, H. Fousse
}

\section{To cite this version:}

R. Mainard, J. Kleinclauss, H. Fousse. Calorimètre adiabatique à température variable et à surfaces d'échanges sphériques. Revue de Physique Appliquée, 1970, 5 (6), pp.823-828. 10.1051/rphysap:0197000506082300 . jpa-00243460

\section{HAL Id: jpa-00243460 https://hal.science/jpa-00243460}

Submitted on 1 Jan 1970

HAL is a multi-disciplinary open access archive for the deposit and dissemination of scientific research documents, whether they are published or not. The documents may come from teaching and research institutions in France or abroad, or from public or private research centers.
L'archive ouverte pluridisciplinaire HAL, est destinée au dépôt et à la diffusion de documents scientifiques de niveau recherche, publiés ou non, émanant des établissements d'enseignement et de recherche français ou étrangers, des laboratoires publics ou privés. 


\title{
CALORIMẼTRE ADIABATIQUE A TEMPÉRATURE VARIABLE ET A SURFACES D'ÉCHANGES SPHÉRIQUES
}

\author{
par R. MAINARD, J. KLEINCLAUSS et H. FOUSSE \\ Institut de Physique, 2, rue de la Craffe, 54, Nancy
}

(Reçu le 26 juin 1970)

\begin{abstract}
Résumé. - Un nouveau type de calorimètre adiabatique a été réalisé. Les valeurs absolues des chaleurs spécifiques peuvent être mesurées de façon directe et la précision facilement appréciée. Pour aboutir à ce résultat, il a fallu surmonter deux types de difficultés. Au point de vue technologique, nous avons été amenés à réaliser des enroulements à spires jointives sur des surfaces sphériques. Au point de vue expérimental, nous avons dû éliminer les fuites thermiques par conduction le long des différents fils conducteurs partant du calorimètre. Cet appareil est destiné à la mesure de chaleurs spécifiques de substances antiferromagnétiques à basse température.
\end{abstract}

Abstract. - A new-model of adiabatic calorimeter has been designed. Absolute values of specific heats can be measured directly and the accuracy can be easily determined. To reach that result, two types of difficulties had to be overcome. From the technological point of view, we were led to realize adjacent spiral scrolls on spherical surfaces. From the point of view of the experiment itself, we had to eliminate the thermal losses created by conduction along the wires emerging from the calorimeter. This apparatus is designed for measurements of the specific heats of antiferromagnetic substances at low temperatures.

Introduction. - La plupart des calorimètres actuellement utilisés ont une forme cylindrique. Leur principal avantage réside dans la relative facilité de réalisation, mais leur symétrie imparfaite est souvent préjudiciable à des mesures absolues de chaleurs spécifiques. Ils nécessitent en outre l'utilisation de plusieurs thermocouples.

L'équilibre thermique est assez long à obtenir, ce qui, aux dires de certains auteurs [1], est une cause de l'imprécision des mesures.

Par suite, dans la plupart des cas, il faut se contenter de mesures relatives faisant intervenir la chaleur spécifique d'une substance de référence.

Aussi avons-nous conçu puis réalisé un calorimètre susceptible de mesurer la valeur absolue de la chaleur spécifique de très faibles masses de produits (de l'ordre de quelques grammes). Ce calorimètre possède des surfaces d'échanges sphériques concentriques soigneusement dorées.

Pour contrôler l'appareil, nous avons mesuré la chaleur spécifique entre $0^{\circ}$ et $100^{\circ} \mathrm{C}$ de quelques substances qui avaient été étudiées par de nombreux auteurs : polytétrafluoroéthylène (Téflon) [1] [2] [3], aluminium [4], oxyde de cobalt $(\mathrm{CoO})$ [5] [6].

Nos résultats sont en bon accord avec ceux publiés antérieurement. Cet appareil est destiné ultérieurement à des mesures de chaleurs spécifiques de substances antiferromagnétiques aux très basses températures.

Réalisation du récipient calorimétrique (Fig. 1 et 2). -- Il est formé de deux parties démontables main-
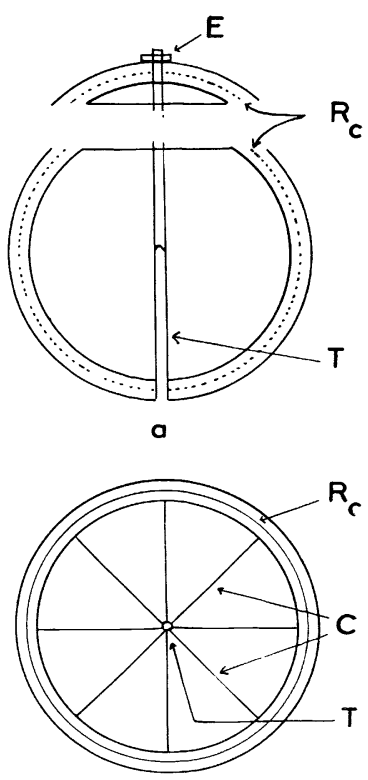

b

FIG. 1. - Le récipient calorimétrique

$a$ : coupe verticale

$b$ : coupe horizontale

tenues ensemble à l'aide d'un écrou de fixation $E$. L'une est une petite calotte qui sert de couvercle, l'autre, beaucoup plus grande, sera remplie de la substance à étudier. Lorsque les deux parties sont fixées ensemble, elles forment une sphère parfaite 


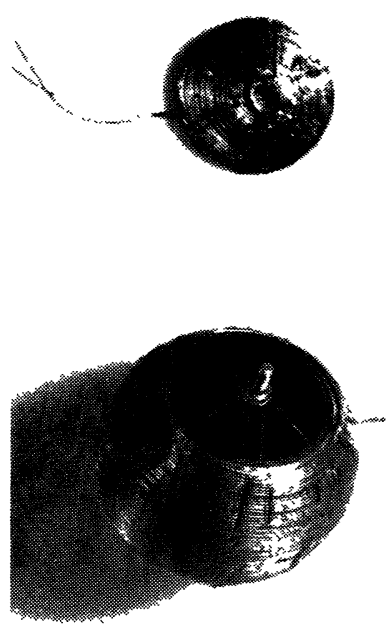

Fig. 2. - L'enroulement chauffant du récipient calorimétrique.

de $2 \mathrm{~cm}$ de diamètre dont la paroi extérieure a été dorée afin de réduire au minimum le facteur total d'émission $(\varepsilon=0,018$ [7] [8]).

Chaque partie du récipient calorimétrique se présente alors sous l'aspect de deux portions de sphères concentriques en cuivre de rayons très voisins, entre lesquelles est bobiné à spires jointives un enroulement chauffant $R_{\mathrm{c}}$ réalisé en fil de constantan de $0,16 \mathrm{~mm}$ de diamètre. Le flux de chaleur qui pénètre à l'intérieur de la sphère est radial et sa valeur est constante par unité de surface de celle-ci. Les différentes parties métalliques ainsi que l'élément chauffant sont collés à l'aide du vernis isolant oléosynthétique $\mathrm{n}^{0} 03.28$ Orange de l'Usine Diélectrique de Delle.

Un petit tube en laiton $T$, fixé sur la sphère, permet d'introduire en son centre un thermocouple destiné à repérer la température de la substance étudiée.

Le récipient calorimétrique est garni intérieurement de cellules de feuilles de cuivre en nid d'abeille $C$ destinées à faciliter les échanges thermiques afin que la température s'uniformise rapidement.

Enceintes adiabatiques. - Le calorimètre est placé au centre d'un ensemble de deux enceintes adiabatiques.

L'enceinte adiabatique intérieure est une sphère concentrique au calorimètre tandis que l'enceinte adiabatique extérieure est un cylindre.

L'enceinte adiabatique sphérique (Fig. 3 et 4) est une sphère dorée intérieurement, de $5 \mathrm{~cm}$ de diamètre, découpée en deux hémisphères; elle porte également sur sa face externe un enroulement chauffant $R_{\mathrm{e}-1}$ réalisé à spires jointives.

Un thermocouple différentiel TD-1, dont une des soudures est placée sur le calorimètre et l'autre sur la face interne de cette enceinte adiabatique permet de s'assurer que les deux sphères sont à la même température. Le récipient calorimétrique est suspendu par un fil $F$ à l'intérieur de l'enceinte et soigneusement

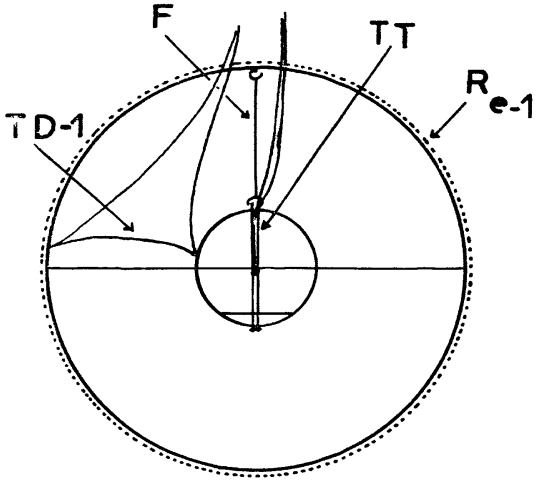

FIG. 3. - Coupe d'ensemble de l'enceinte adiabatique intérieure et du calorimètre.

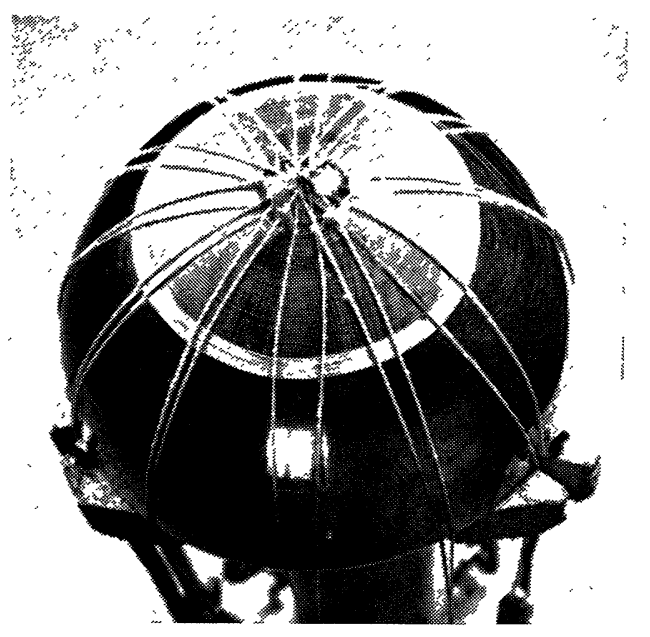

Fig. 4. - Réalisation de l'enroulement chauffant sur un des deux hémisphères constituant l'enceinte adiabatique intérieure.

centré ; par suite, la symétrie de l'appareil nous autorise à n'utiliser qu'un seul couple.

Pour éliminer les pertes calorifiques par conduction le long des fils qui partent du calorimètre, l'ensemble des deux sphères est placé dans une enceinte adiabatique cylindrique fermée à ses deux extrémités. Ce cylindre en laiton de $50 \mathrm{~cm}$ de longueur et de $7 \mathrm{~cm}$ de diamètre, porte extérieurement un enroulement chauffant $R_{\mathrm{e}-2}$. Un deuxième thermocouple différentiel TD-2 permet de s'assurer que le cylindre est à la même température que les deux sphères.

Enceinte métallique extérieure à double paroi (Fig. 5). - Tout cet ensemble est placé dans un dewar métallique à double enveloppe dans lequel règne un vide poussé, de l'ordre de $5 \times 10^{-6}$ torr mesuré à l'aide d'une jauge ionique placée à la partie supérieure de l'appareil.

Cette enceinte est elle-même placée dans un dewar rempli de glace fondante ce qui permet de commencer les mesures à une température voisine de $0^{\circ} \mathrm{C}$.

Montages électriques. - Trois circuits indépendants sont reliés, l'un au calorimètre (circuit de 


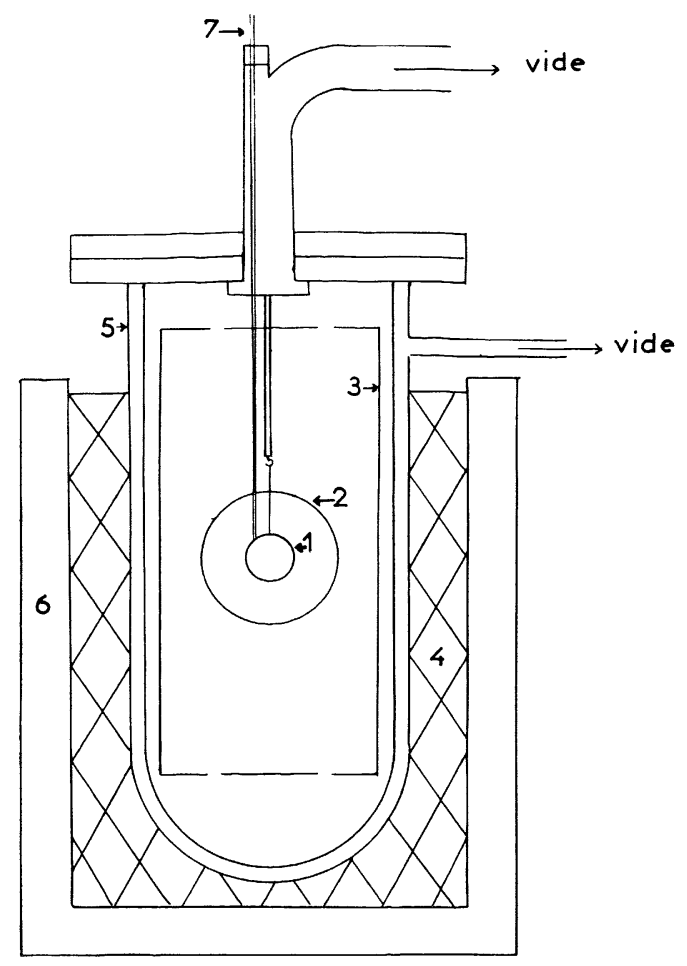

FIG. 5. - Schéma d'ensemble de l'appareil.

1. - Calorimètre.

2. - Enceinte adiabatique intérieure.

3. - Enceinte adiabatique extérieure.

4. - Glace.

5. - Enceinte métallique à double paroi.

6. - Dewar.

7. - Fils de chauffage et de thermocouples.

chauffage), et chacun des deux autres à une des enceintes adiabatiques (circuits de régulation).

Le circuit de chauffage (Fig. 6) comprend une alimentation $A S$, stabilisée en courant à $2 \%$, qui envoie un courant connu $i_{\mathrm{c}}$, rigoureusement constant, dans la résistance chauffante du calorimètre $\left(R_{\mathrm{c}}=170 \Omega\right)$. Il nous est indispensable de connaître, avec le maximum de précision, la valeur de l'énergie envoyée au calorimètre pendant un temps donné. Contrairement au courant $i_{\mathrm{c}}$ qui reste constant, la tension $V_{\mathrm{c}}$ aux bornes de la résistance $R_{\mathrm{c}}$ du calorimètre est susceptible de varier en fonction de la température de $R_{\mathrm{c}}$. Nous avons donc utilisé un appareil intégrateur IG qui nous permet de connaitre $\int V \mathrm{dt}$ aux bornes d'une résistance pendant un temps quelconque, $V$ pouvant varier en fonction du temps.

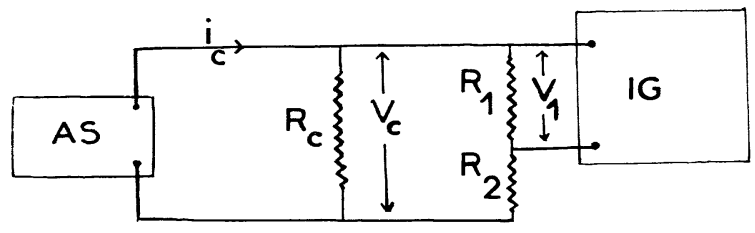

Fig. 6. - Circuit de chauffage.
Cet intégrateur, ne pouvant supporter une tension $V$ supérieure à 0,5 volt, ce qui correspondait à une intensité $i_{\mathrm{c}}=3 \mathrm{~mA}$ beaucoup trop petite, n'a pas été branché directement aux bornes de $R_{\mathrm{c}}$, mais à celles de la résistance $R_{1}$ d'un montage en diviseur de tension. Les deux résistances $R_{1}$ et $R_{2}$ ont une très grande valeur (par exemple $R_{1}=10^{4} \Omega$ et $R_{2}=9 \times 10^{4} \Omega$ ) et ne modifient pas sensiblement la valeur du courant $i_{\mathrm{c}}$ ainsi que nous avons pu le vérifier au cours de l'étalonnage préalable de l'intégrateur.

L'expression de l'énergie fournie au calorimètre est alors :

$$
W=i_{\mathrm{c}}\left(\frac{R_{1}+R_{2}}{R_{1}}\right) \int V_{1} \mathrm{dt}
$$

où $V_{1}$ est la tension aux bornes de la résistance $R_{1}$ et $\int V_{1} \mathrm{dt}$ la valeur indiquée par l'intégrateur.

Compte tenu des erreurs d'étalonnage et de lecture des différents appareils, l'incertitude sur le résultat de la mesure de l'énergie envoyée au calorimètre est inférieure à $1 \%$ comme le montre un calcul d'erreur élémentaire.

Un thermocouple TT cuivre-constantan dont une soudure pénètre au centre du calorimètre, l'autre étant maintenue à $0^{\circ} \mathrm{C}$, est relié à un pont de mesure indiquant le $1 / 10^{\mathrm{e}}$ de microvolt et donne la température précise du calorimètre et de son contenu. En effet, après avoir vérifié que notre thermocouple donnait des tensions identiques à celles de l'étalonnage international [9], nous avons établi une équation du $3^{\mathrm{e}}$ degré donnant la température en fonction aux de la tension bornes du thermocouple et valable pour l'intervalle $0-100^{\circ} \mathrm{C}$. Cette équation :

$\Theta=0,037 \times 10^{9} V^{3}-0,77 \times 10^{6} V^{2}+26 \times 10^{3} V$

où $\Theta$ est la température exprimée en degrés Celsius et $V$ la tension correspondante exprimée en volts, nous permet de connaître l'élévation de température du calorimètre et de son contenu avec une incertitude inférieure à $6 \%$.

Le deuxième circuit (Fig. 7) a pour but de maintenir la surface externe du calorimètre et la surface interne

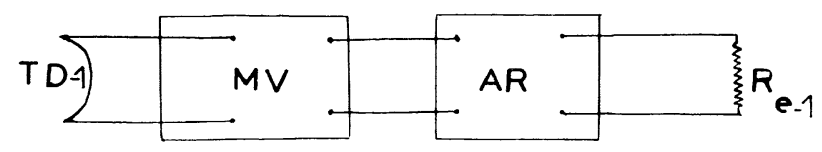

FIg. 7. - Circuit de régulation.

de l'enceinte adiabatique sphérique à la même température. Le thermocouple différentiel TD-1 cuivreconstantan indique la différence de température entre les deux surfaces. Lorsque celle-ci est différente de zéro, le microvoltmètre $\mathrm{MV}$, très sensible $(1 \mu \mathrm{V}$ pleine échelle), amplifie la tension donnée par le thermocouple différentiel et transmet une information à un amplificateur-régulateur AR que nous avons fait réaliser 
spécialement pour notre montage. Celui-ci envoie à son tour un courant plus ou moins intense dans la résistance $R_{\mathrm{e}-1}=400 \Omega$ qui entoure l'enceinte adiabatique sphérique. Dans ces conditions, la différence de température entre les deux surfaces est au maximum de $0,003^{\circ} \mathrm{C}$ ce qui correspond à une différence de potentiel de $0,1 \mu \mathrm{V}$.

Le troisième circuit qui permet de maintenir l'enceinte adiabatique cylindrique à la même température que l'enceinte adiabatique sphérique est identique au deuxième circuit.

Principe de la mesure. - Au cours d'une première expérience, nous fournissons au calorimètre vide une certaine énergie $W_{0}$. Nous coupons le chauffage du calorimètre et nous attendons que sa température se stabilise, ce qui se produit bien avant les 10 minutes que dure cet arrêt. Nous notons la différence de température $\delta \Theta_{0}$ entre le début du chauffage et la fin de la période de stabilisation. Nous en déduisons la capacité calorifique du calorimètre vide :

$$
C_{0}=\frac{W_{0}}{\delta \Theta_{0}} .
$$

Nous répétons ces opérations pour chaque intervalle correspondant à une élévation de température $\delta \Theta_{0}$ de $1^{\circ} \mathrm{C}$ environ. Ces mesures nous permettent de tracer la courbe représentative de la variation de la capacité calorifique du calorimètre vide en fonction de la température (Fig. 8).

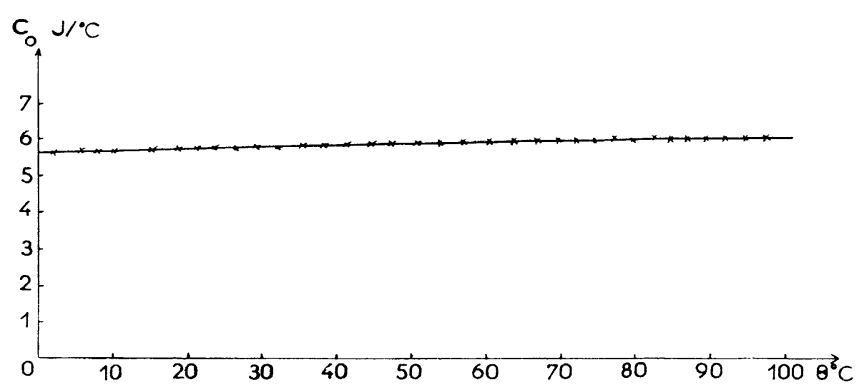

FIG. 8. - Capacité calorifique du calorimètre vide.

Nous effectuons les mêmes opérations lorsque le calorimètre est rempli d'une masse $m$ de la substance à étudier. Nous obtenons de nouvelles valeurs $W_{1}$ de l'énergie envoyée au calorimètre plein et $\delta \Theta_{1}$ de l'élévation de température correspondante.

Nous en tirons la capacité calorifique du calorimètre plein, soit $C_{1}=W_{1} / \delta \Theta_{1}$ et la courbe représentative de la variation de celle-ci en fonction de la température (Fig. 9a, 10a, 11a).

On déduit de ces deux séries de mesures la chaleur spécifique de la substance étudiée, soit : $C_{\mathrm{p}}=\left(C_{1}-C_{0}\right) / m$ en $\mathrm{J} / \mathrm{g}{ }^{\circ} \mathrm{C}$, où $C_{1}$ et $C_{0}$ sont respectivement les capacités calorifiques du calorimètre plein et du calorimètre vide à la même température, ainsi que les courbes représentant la variation de cette cha- leur spécifique en fonction de la température (Fig. 9b, $10 b, 11 b)$.

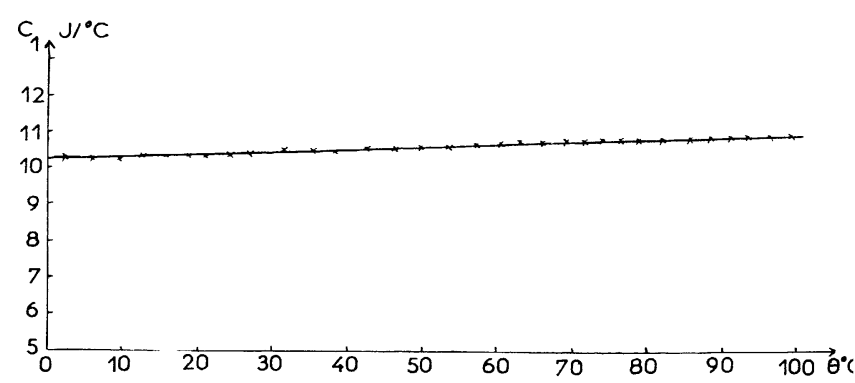

FIG. 9a. - Capacité calorifique du calorimètre rempli d'Aluminium.

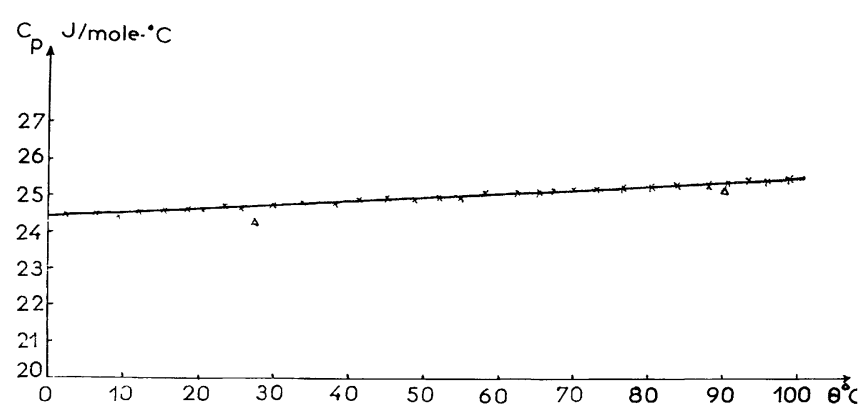

FIG. $9 b$. - Chaleur spécifique de l'aluminium $\times$ aluminium en poudre, ce travail

$\triangle$ bloc d'aluminium, McDonald [4]

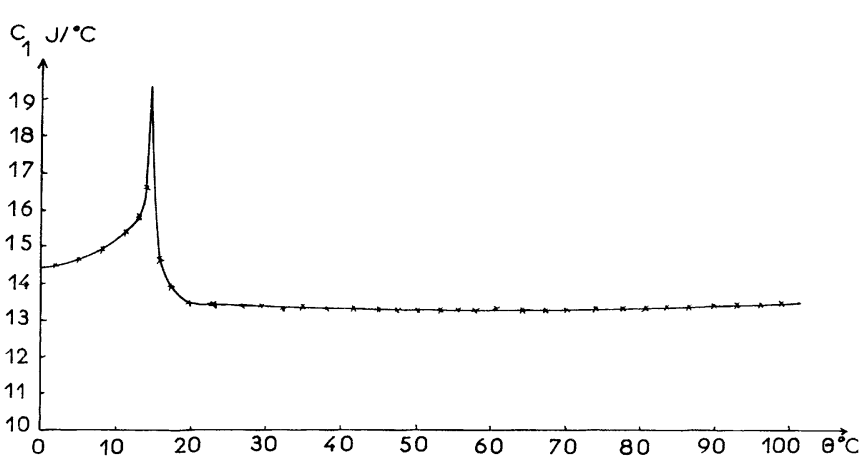

FIG. 10a. - Capacité calorifique du calorimètre rempli de CoO.

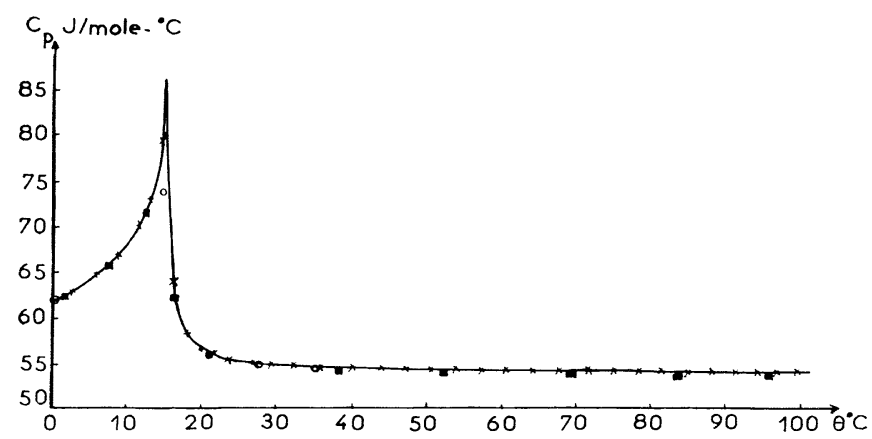

FIG. 10b. - Chaleur spécifique de $\mathrm{CoO}$

$\times$ ce travail

E. G. King [5]

- R. Mainard [6] 


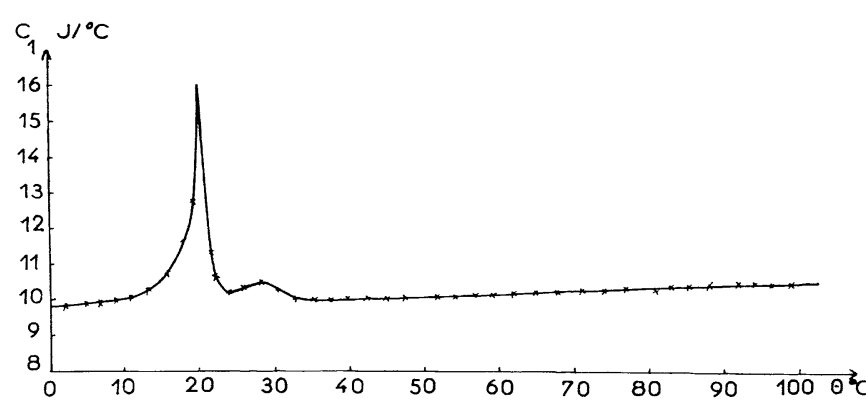

FIG. 11a. - Capacité calorifique du calorimètre rempli de Polytétrafluoroéthylène.

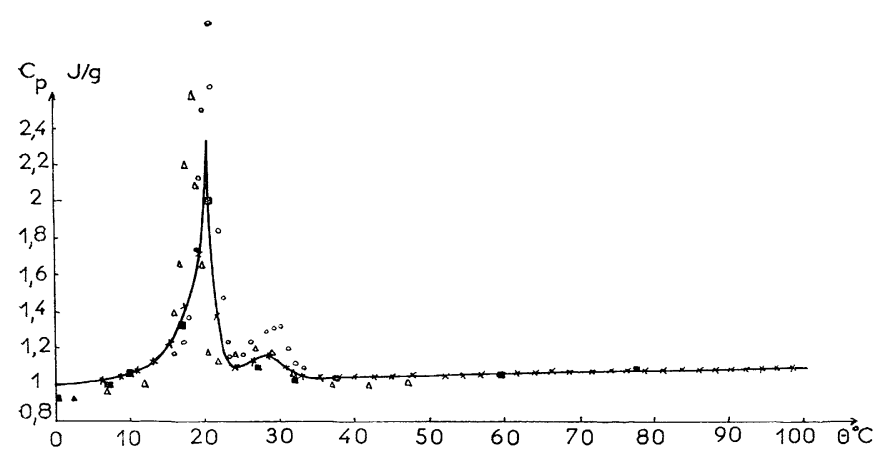

FIG. $11 b$. - Chaleur spécifique du P T F E (Téflon)

$X$ en copeaux, ce travail

$O$ en bloc, Findenegg et coll. [1]

$\triangle$ en poudre, Marx et Dole [2]

moulé, Furukawa et coll. [3]

Précision des mesures. - La précision des résultats est fonction, d'une part des erreurs introduites par les différents appareils, d'autre part des pertes calorifiques qui pourraient éventuellement être dues à des fuites thermiques par rayonnement, par convection et par conduction.

Nous avons vu précédemment que les premières ont été calculées et trouvées inférieures à $1 \%$.

Le flux des pertes par rayonnement est donné par l'équation de Christiansen [10] [11] valable pour des sphères concentriques :

$$
\Phi_{12}=\frac{1}{1+\varepsilon_{1}\left(\frac{1}{\varepsilon_{2}}-1\right) \frac{A_{1}}{A_{2}}} \varepsilon_{1} \sigma A_{1}\left(T_{1}^{4}-T_{2}^{4}\right)
$$

où $A_{1}$ et $A_{2}$ sont les surfaces d'échange, $\varepsilon_{1}$ et $\varepsilon_{2}$ les facteurs d'émission correspondants, $T_{1}$ et $T_{2}$ les températures absolues et $\sigma$ la constante de Stefan $(*)$.

Or les températures $T_{1}$ et $T_{2}$ ne diffèrent que de $0,003{ }^{\circ} \mathrm{C}$ au maximum ce qui correspond à un flux $\Phi_{12}=1 \mu \mathrm{J} / \mathrm{s}$, soit à une erreur de $0,1 \%$ sur la mesure de l'énergie.

Les pertes par convection ont été éliminées grâce au vide poussé obtenu dans tout l'ensemble.

(*) Des travaux récents font état de recherches concernant le transfert par rayonnement entre sphères concentriques entre lesquelles se trouve un gaz [12] [13].
Les pertes par conduction le long des fils qui pénètrent dans l'enceinte adiabatique sphérique ont été rendues négligeables par la présence de l'enceinte adiabatique cylindrique.

Avec toutes ces précautions, nous avons noté que, si nous abandonnons l'ensemble calorimètre-enceintes adiabatiques à une température donnée, cette dernière ne variait au maximum que de $0,2^{\circ} \mathrm{C}$ en 12 heures soit une erreur de $0,1 \%$ sur la mesure de l'énergie.

Compte tenu de la somme de toutes ces erreurs, nous pouvons être assurés que la précision du résultat est meilleure que $2 \%$.

Résultats. - Les mesures de chaleurs spécifiques qui ont été réalisées sur des substances précédemment étudiées, ont conduit à des résultats en bon accord avec ceux publiés antérieurement.

Il existe certains écarts entre les résultats donnés par différents auteurs mais il faut remarquer que les substances étudiées sont souvent d'origines différentes et ne se présentent pas sous la même forme.

Ainsi pour l'aluminium, McDonald [4] avait mesuré la chaleur spécifique d'un bloc de $\mathbf{6} \mathrm{g}$ environ alors que nous avons rempli notre calorimètre avec $5,0987 \mathrm{~g}$ de poudre d'aluminium obtenue à partir d'un bloc très pur $(99,99 \%)$.

Pour l'oxyde de cobalt, nous retrouvons pratiquement les résultats antérieurs de King [5] et Mainard [6] car, dans tous les cas, l'oxyde de cobalt étudié était sous forme d'une poudre polycristallisée.

Enfin le polytétrafluoroéthylène a été étudié sous de nombreuses formes: bloc, copeaux, poudre, moulé. Nous avons utilisé des copeaux et nos résultats, quant à la valeur absolue de la chaleur spécifique et à la température d'anomalie, sont très proches de ceux publiés par Furukawa, McCoskey et King [3] (moulé). On peut remarquer que la température d'anomalie que nous trouvons pour les copeaux $\left(20^{\circ} \mathrm{C}\right)$ se situe entre celle donnée par Findenegg, Wilhelm et Kohler [1] pour le bloc $\left(20,5^{\circ} \mathrm{C}\right)$ et celle donnée par Marx et Dole [2] pour la poudre $\left(19^{\circ} \mathrm{C}\right)$.

Conclusion. - Notre appareil, s'il est de réalisation technologique relativement délicate, est d'un emploi facile. L'utilisation d'un seul thermocouple entre les surfaces d'échanges sphériques rend, en effet, facile et rapide le démontage de l'appareil entre deux séries de mesures.

Par ailleurs, des mesures très précises peuvent être faites sur des quantités de substances très petites.

Les fuites thermiques sont éliminées de façon satisfaisante, ce qui permet, lors d'une mesure, d'une part de supprimer toute erreur inhérente au transfert de chaleur parasite entre les surfaces d'échanges sphériques lors de l'échauffement de la substance, et d'autre part d'attendre le temps nécessaire à l'établissement de l'équilibre thermique.

Enfin, cet appareil pourra être utilisé sans transformations fondamentales aux très basses températures. 


\section{Bibliographie}

[1] FindenegG (G. H.), Wilhelm (E.) und Kohler (F.) Mh. Chem., 1966, 97, 94.

[2] Marx (P.) and Dole (M.), J. Amer. Chem. Soc., 1955, 77, 4771.

[3] Furukawa (G. T.), McCoskey (R. E.) and KING (G. J.), J. Res. Nat. Bur. Stand., 1952, 49, 273.

[4] McDonald (R. A.), J. Chem. Eng. Data, 1967, 12, 115.

[5] KING (E. G.), J. Amer. Chem. Soc., 1957, 79, 2399.

[6] Mainard (R.), Thèse, Nancy, 1963.

[7] Schmidt (H.) und FurthmanN (E.), Mitt. KaiserWilhelm-Instit. Eisenforsch. Dusseldorf, Abhandl., 1928, 109, 225.
[8] McAdams (M. H.), Transmission de la chaleur, p. 528 , Dunod, 1961.

[9] American Institute of Physics Handbook, p. 411, 1963.

[10] Christiansen (C.), Wiedemanns Ann., 1883, 19, 267.

[11] Jаков (Max), Heat Transfer, II, 5, John Wiley and Sons, 1957.

[12] Sparrow (E. M.), Usikin (C. M.) and Hubbard (H. A.), J. Heat Transfer, Trans Asme, 1961, C. 83, 199.

[13] Ryhming (I. L.), Int. J. Heat Mass Transfer, 1966, 9, 315 . 